\title{
Expression of Notch-1 receptor and its ligands Jagged-1 and Delta-1 in amoeboid microglia in postnatal rat brain and murine BV-2 cells.
}

\begin{abstract}
Notch-1 receptor signaling pathway is involved in neuronal and glial differentiation. Its involvement in microglial functions, however, has remained elusive. This study reports the localization of Notch-1 receptor immunoreactivity in the amoeboid microglial cells (AMC) in the postnatal rat brain. By immunofluorescence, Notch-1 receptor was colocalized with its ligands, Jagged-1 and Delta-1, in the AMC in the corpus callosum and subventricular zone. Notch-1 immunopositive cells were confirmed to be microglia labeled by OX42 and lectin. Immunoexpression of Notch-1 receptor was progressively reduced with age. Western blot analysis showed that Notch-1 protein level in the corpus callosum in which the AMC were heavily populated was concomitantly decreased. In postnatal rats challenged with lipopolysaccharide (LPS), Notch-1 receptor immunofluorescence in AMC was noticeably enhanced. Furthermore, Notch-1 protein level in the corpus callosum was increased as revealed by Western blotting analysis. In primary microglial culture treated with LPS, mRNA expression of Notch-1 and its ligand Jagged-1 was upregulated but that of Delta-1 was reduced. The expression pattern of Notch-1 and its ligands was confirmed in murine BV-2 cells. Furthermore, Notch-1 neutralization with its antibody reduced its protein expression. More importantly, neutralization of Notch-1 concomitantly suppressed the mRNA expression of IL-6, IL-1, M-CSF, and iNOS; TNF- $\alpha$, mRNA expression, however, was enhanced. Western blot confirmed the changes of protein level of the above except for IL-6, which remained relatively unaltered. It is concluded that Notch-1 signaling in the AMC and LPSactivated microglia/BV-2 cells modulates the expression of proinflammatory cytokines and nitric oxide.
\end{abstract}

Keyword: Cytokines; Ligands; Microglia; Nitric oxide synthase; Notch-1. 